Irene Roozen* and Michel Meulders

\title{
Has TV advertising lost its effectiveness to other touch points?
}

DOI 10.1515/commun-2015-0019

\begin{abstract}
In this paper we analyze the relative effectiveness of the moment of contact (touch point) between a brand and an individual consumer. The concept of effectiveness is made operational through the use of both attitude (cognitive, emotional and conative) and awareness measures (recall and recognition). The main research uses a $4 \times 4$ Latin square confounded within subjects factorial design with different touch points and brands. The appropriate stimuli were identified in a preliminary study. The results indicate that, overall, TV advertising and print advertisements - the traditional media channels - are significantly more effective than the internet, a non-traditional media channel.
\end{abstract}

Keywords: advertising, touch point, effectiveness, attitude, awareness

\section{Introduction}

The sustained rise of the internet not only introduces new marketing communication channels but also increases the challenge for marketing managers to select appropriate media channels (Danaher and Rossiter 2011). In addition, while the number of available media channels has increased, media budgets have remained almost the same (Danaher and Rossiter 2011).

According to recent data from the Duke University Survey, spending on traditional media (television, radio and print) will decline while, according to 468 U.S. chief marketing officers, the internet - for example, social media spending - is expected to increase to $11.5 \%$ of the total advertising budgets in 2013 and is estimated to reach $21.3 \%$ in the next five years (Moorman 2013). In 2013, the United States spent around \$140.2 billion on advertising. Internet advertising expenditure grew by $15.7 \%$ between 2012 and 2013 while that in television declined by $0.1 \%$, in radio declined by $5.6 \%$, and in newspapers declined by $3.7 \%$. Magazine advertising increased by $1.8 \%$ (Kantar Media, http://www.kantarmediana.com/news/03172010.htm).

*Corresponding Author: Irene Roozen, KULeuven, E-mail: irene.roozen@kuleuven.be Michel Meulders, KULeuven, E-mail: michel.meulders@kuleuven.be 
While the choice between the different (non-)traditional media channel options is more and more a challenge for marketing officers, the relative advertising effectiveness of the different media channel options, touch points, has not been established. It is, therefore, not straightforward to combine them in an integrated marketing communication (IMC) framework which aims at distributing persuasive messages for building (sustainable) relationships with customers (Lin, Venkataraman, and Jap 2013). Moreover, to date, no study has comprehensively examined the relative advertising effectiveness of different media channel options for the same brand by asking respondents to evaluate the different key media channels individually.

\section{Review of the literature}

In general, touch points are defined as points of communication or moments of contact between an organization or brand and an individual consumer or stakeholder (Jenkinson 2007). The viewing by potential consumers of advertisements for a product or a brand on different media channels is a key example of such touch points. Clearly, the different media channels work in different ways and, therefore, create different 'touch points'. Factual differences between media instruments are, for example, measures of reach, frequency, share-ofvoice, and cost-per-point. Moreover, differences between touch points can also be assessed on the basis of various evaluation metrics: for example, the attitude toward the advertisement, the attitude toward the brand or product, purchase intention (PI), the value to the brand and the awareness of the advertisement (i.e., recall and recognition) (Danaher and Rossiter 2011; Hallward 2008; Jenkinson 2007). Moreover, the creative aspects of the advertisement, the interaction possibilities, and the emotions differ between the different media instruments and touch points, which renders the planning of marketing communication complicated (Jenkinson 2007).

The effectiveness of different touch points as revealed by the attitude toward different advertisements for the same brand has not been investigated for real-life advertisements in different media in a simultaneous manner. Attitudes that are formed toward an advertisement through a touch point can influence consumers' attitudes toward the product/brand and/or their intention to purchase the product/brand (Goldsmith and Lafferty 2002; Homer 1990; MacKenzie, Lutz, and Belch 1986). Therefore, the ultimate goal of creating a touch point through an advertisement is to form positive attitudes towards the brand and the product toincrease the likelihood that consumers will buy the product 
and/or the brand. Furthermore, research shows that positive emotional responses to an advertisement and responses of liking an advertisement are also positively related to purchase intentions and to the consumers' ability to recall the advertised brand (Dubow 1994). Consumers with a positive attitude toward an advertisement are more able to recall the advertisement than their counterparts. Goldsmith and Lafferty 2002 found 'in general' more recalled advertisements from TV than from internet, radio and print touch points. However, their results were based on a free recall protocol (respondents were asked to indicate liked and disliked advertisements along with the medium of all kinds of brands and medium channels). They also indicate that research should ideally use representative samples and multiple measures with additional variables (with more items) in an experimental setting covering the same brands in different media to better assess the relationship between attitude and recall (Goldsmith and Lafferty 2002).

Schultz, Cole, and Bailey 2004 suggest that the return-on-touch-point-investment is the most relevant way to measure the returns of marketing communications. They suggest that this concept can be measured by starting an analysis of the purchase dynamics of the customer/group with the brand and with competitors, so that in the end different classifications of customers can be made (e.g., 'retention', 'grow loyalty', 'grow penetration'). Furthermore, the role of the different touch points in creating value in the consumer-brand relation has to be estimated. The value of an individual touch-point is related to the value of an individual interaction and is probably based on the value of the customers or prospects it involves, the degree to which the event (the touchpoint) has the ability to influence their attitudes, behavior, and numerous other factors (Schultz et al. 2004). However, for some touch-points, the interaction is anonymous in nature and therefore difficult to take into account whereas for other touch-points the frequency plays a significant role. This suggests that it is very difficult to set up an experiment in which all relevant touch point characteristics are taken into account.

The 'top of mind' awareness of consumers, that is, consumers' ability to recall the advertised brand, is another component of advertising effectiveness (Goldsmith and Lafferty 2002). Research suggests that consumers with a positive attitude toward the advertisement are significantly better able to recall the advertisement (and indirectly the brand/product) than those with a negative attitude (Goldsmith and Lafferty 2002). Bruner and Kumar 2000 found in their experimental setting that the attitudes toward the website were positively related to the attitude toward the advertisement, the brand and the intentions to purchase the product. 
To assess the effectiveness of advertising on the internet, most marketing managers are focusing on the number of visitors, the clicks and the demographic characteristics of the visitors (Green and Elgin 2001; Yoon and Kim 2001). However, click-through rates on banner advertisements, for example, were $30 \%$ in 1994 and only 0.3\% in 2000 (Green and Elgin 2001). Goldsmith and Lafferty 2002 found that the attitude-toward-the-site is an important measure of the effectiveness of advertising on the internet. They found that "liking" a web site and "liking an advertisement" influence other related advertisement evaluations, for example, brand recall or attitude toward the brand. Nagar 2009 analyzes the responses of customers toward the internet and television as a medium of advertising. He asked the participants to evaluate advertisements on the internet and TV and found that respondents considered the internet as more effective in terms of informativeness, whereas TV was more effective in catching the consumer's attention and in increasing the likelihood of purchase of the product after exposure. However, no differences were found between the emotional content and the entertainment capability of the two touch points. In Nagar's study 2009, advertisements on TV and the internet were investigated. However, no experimental setting with specific brands and/or products was designed for the data collection. Moreover, the definition of internet advertising in Nagar's study 2009 is very broad, for instance, banner advertisements, popups, pop- unders, interstitials. He proposed that an experimental design with more advertising media channels (touch points) would, therefore, be more appropriate for comparing the advertisements.

Companies are increasingly using cohesive messages across multichannel media options which are consistent with an integrated marketing communication philosophy (Lin et al. 2013). They experiment with cross-media advertising strategies such as broadcasting television commercials, along with traditional print advertisements, radio advertisements and videos on the company's website. However, there is almost no research using individual consumer preferences for specific brands and/or products that aids companies when planning their communication strategies across multiple media (Lin et al. 2013).

The model devised by Lin, Venkataraman and Jap 2013 investigates, for example, the effectiveness of consumers' media choices, and analyzes 'joint consumption' scenarios of radio, television, print and internet. Danaher and Rossiter's correspondence analysis 2011 is based on evaluations of only one media channel at a time.

Instead of measuring consumers' attitudes, the sales of a product or of a brand are often used as a measure of the advertising effectiveness of the different touch points (Lin et al. 2013; Naik and Raman 2003). However, in many situations, advertising does not directly influence sales but impacts on interme- 
diary variables related to branding or communications, which, in turn, can affect sales after a certain time. Significant and variable time lags might, therefore, occur, which can significantly bias research results (Hallward 2008). Moreover, sales data are influenced by a great number of other variables (e.g., developments in disposable income, changes in relative prices, emergence of competing or complementary products) which are unrelated to advertising campaigns. However, sales data are often available and this might explain why they are often used in measuring and estimating advertising effectiveness.

McKenzie and Royne 2009 state that only a small group of specialized marketing agencies take account of the relative advertising effectiveness of different touch points when planning their communication. This suggests that the concept of communication planning - touch-point planning - is still in its infancy. Dahlén and Edenius 2007 investigate how consumers identify traditional and non-traditional advertising media. Their research results show that for the different touch points, the persuasive intent of an advertisement message is significantly lower when it is placed in non-traditional compared to traditional advertising; whereas the advertisement's credibility and the attitude towards the message are significantly higher in non-traditional media. However, the perceptions and classifications of media as well as the advertising were not taken into account.

In conclusion, while the literature contains a number of studies that have analyzed the effectiveness of traditional and non-traditional advertisements, there remain important gaps in our knowledge. First, research is typically based on sales figures, numbers of visitors of websites, or free recall protocol measurements rather than on consumers' attitudes and/or awareness of the different touch points. Second, no comparisons of different touch points for the same brands are analyzed in a simultaneous manner while controlling for other factors. Third, no 'real products/brands' and 'real advertisements' are analyzed for the different touch points.

Given these methodological limitations, it can be questioned whether the available research provides reliable guidance as to the optimal allocation of marketing budgets across different media channel options. In particular, research results do not unequivocally indicate whether the attitudes toward the advertisements on traditional media channels (TV, radio, print) are relatively more or less positive than the attitude toward advertisements on the internet.

To address these shortcomings, this study is based on an experimental design in which we compare a small sample of touch points for different brands from different product categories in a simultaneous fashion. We rely on 'real products/brands' and 'real advertisements' that were used in Belgium during the period March to December 2009. The consumers' attitude toward the adver- 
tisement - for cognitive, emotional and conative aspects - and their awareness of the brand (recall and recognition) were measured as an indication of the advertising effectiveness of the different touch points. To increase the external validity of our research results, we have used real-life (brand) campaigns and/ or messages which were broadcast at the same time on different media during a certain period.

Against the background of our experimental design, which addresses many of the shortcomings of previous literature, our research questions concern the effectiveness of the different touch points (for the same brands simultaneously) and, in particular, the comparison of traditional (TV, radio, print) touch points versus non-traditional (internet) touch points for the same brands.

[RQ1] Are there significant differences between the attitudes (cognitive, emotional and conative) towards the touch points for the different brands considered in our study?

[RQ2] Are there significant differences between the awareness scores of the different touch points?

Given that differences in attitude and awareness are likely to be influenced by the type of brands studied, the target groups considered and the nature of the advertisement, the answers to the research questions are obviously limited to our sample context. However, as argued above, given the need to include many control variables and to test different touch points simultaneously in a robust experimental design, it seems very difficult to extend our samples to cover more products, audiences and advertisements since this would lead to an unwieldy data set. This, in turn, suggests that our research would have to be replicated for other settings in order to draw more generalizable answers to the research questions.

\section{Overview of this study}

\subsection{Research design}

As discussed in the literature review, the effectiveness of advertising and the effectiveness of touch points can be measured in different ways using different types of data collection. In this research we opt for the relative effectiveness of different touch points for the same brands. More specifically, touch points will be compared on the basis of different attitude scales (RQ1): cognitive attitude 
scales (attention, informativeness, general attitude towards the advertisement), emotional scales (entertainment, irritation, emotions) and conative scales (purchase intention).

Subsequently, we will investigate the awareness of the different touch points for the different brands (RQ2). On the bases of the results, marketers can adjust their media budgets and improve their media planning towards the different touch points according to their effectiveness. As a result, the communication or moment of contact between an organization or a brand and an individual consumer or stakeholder can be optimized.

To increase the external validity of the research we selected, in our experimental setting, real-life advertisements of the different touch points from known brands. We opted to select advertisements which were not specially designed for our experiment, and which were also spread across the different touch points simultaneously in Belgium during a concentrated period of time. To select the different brands for our experimental research a preliminary research was conducted. The preliminary research consisted of two parts. In the first study we selected - on the basis of secondary data material - companies who relied simultaneously on the different touch points during a certain period of time. In the second preliminary study we investigated the general knowledge/experience of the different brands and focused on the website experience of the brands separately. We did this to ensure that the websites we selected could actually be considered as having a significant 'advertising' potential and, thereby, could facilitate genuine touch points. We consider this important since websites fulfill many different functions, and not all can be presumed to have the potential to provide touch points through internet 'experiences', for example, perceived interactivity, which respondents would consider comparable to traditional touch points.

Based on the research results of this preliminary research, which led to the selection of the brands/products used for our main research, empirical research was carried out to compare the effectiveness of the touch points (for the traditional media: TV commercials, radio commercials and print advertisements, and 'the website' as non-traditional media instrument). For the empirical research we designed an experiment in which a participant had to evaluate all the different touch points separately. A strong advantage of our experimental setting is the internal control. However, this implies the use of forced exposure which can affect the attitudes toward the advertisements in reality. We try to control for the impact of this limitation on the relative scores of the different touch points by using a within-subject design for the evaluations (i.e., all touch points were affected by the forced nature of the exposure). 


\subsection{Preliminary research}

The goal of the preliminary research was to select the products/brands for our empirical research. The preliminary research consisted of two parts. First, three experts made a pre-selection of available advertisements using all the 'traditional' touch points for the selected period in the Flemish market. This led to the selection of eight brands. Second, the selected brands were analyzed according to their 'experience' and knowledge, and an additional investigation of the website - the 'non-traditional' touch point - used during the selected period, was carried out. This led to the selection of four out of the eight brands, which were subsequently used in our empirical research. The limitation to four brands was necessary in this Latin square factorial design to ensure that all the respondents would view all four touch points for different brands.

\subsection{Preliminary research: Study 1}

Radio and TV spots broadcast on Flemish (commercial) channels for 2009 were investigated (data collection for the empirical research started May 2011). The most important selection criterion of the brands was that the two touch points were used during the same period with the 'same' kind of message about the brand for the radio and TV spot. For the selection of the brands three different experts analyzed the radio and TV spots for 35 different brands. Brands were first classified into tangible (consumer) products and intangible (service industry) products.

Furthermore, we investigated whether the brands also used print advertisements during the same time period. More than 15 companies were contacted to obtain the exposed print advertisements during the same period (unfortunately they were not always available). Four different brand names for the tangible consumer products were selected based on the explorative research done by the three experts on the spots and the print advertisements. All four products belong to the group of fast-moving consumer products, that is, Campina (dairy products), Evian (mineral water), Jules Destrooper (biscuits and cakes) and Brantano (shoes). Also, four different brand names from the service industries (intangible product category) were selected, that is, De Post (national and international mail), Nationale Loterij (the biggest charity lottery organization in Belgium), Thomas Cook (tour operator) and De Lijn (public transport organization in Flanders, the Dutch speaking part of Belgium). For all eight brands, the website of the brand was included as a fourth touch point (all had websites). Finally, for the eight different products, knowledge and experience of the brands were analyzed by the authors in the second pre-test study. 


\subsection{Preliminary research: Study 2}

A further selection among the eight brands was made in the second pre-test study. We investigated 'experience' and 'knowledge' of the selected brands and attitude towards the 'non-traditional touch point' (the website) of the selected brands. Unlike for traditional touch points, measuring the attitude towards the 'non-traditional' touch point (internet) is not straightforward (Karson, McCloy, and Bonner 2006; Nagar 2009). The internet as a touch point, for example, can extend the function of advertising far beyond the possibilities of the traditional touch points. Consumers can obtain information about the product or the service and can also use, for example, the various links about the product or services such as downloads or they can place orders online (Nagar 2009). Advertisements on the internet, compared to TV, radio or print advertisements, can also offer more content about specific features of the service or the product, and the customer can decide what she or he wants to see. The internet can, therefore, act like a sales and distribution channel and allow the customers to interact with the products. This means that 'interactivity' between the product and the customer for the internet, compared to the other 'traditional' touch points, can be very important (Nagar 2009). The application possibilities of the internet (website) and the more traditional media (radio, TV and print advertisements) are significantly different. The function of advertising via the internet can extend far beyond what traditional media are able to accomplish. Especially, interactivity is unique compared with the other mass media instruments (Song and Zinkhan 2008). Newhagen, Corders and Levy 1995 used the term 'perceived interactivity', which is based on the viewers' psychological sense of efficacy as well as on the viewers' sense of the media system's interactivity. For the selection of the brands we, therefore, have taken into account in the second preliminary test the attitude toward the 'interactivity' of the website of the brand.

For the second study, an online questionnaire (with the support of Qualtrics software) was used to collect data. Respondents were asked to go to the website to fill out the questionnaire. The average time to fill out the questionnaire was between 35 and 50 minutes (depending on the 'interactivity' time the respondents spent on the two different websites). In total, 20 respondents filled out the questionnaire (60\% females, $40 \%$ males). The average age of the respondents was 39.6 years (s.d. 14.6 years).

First, the subjects were asked to evaluate eight different brand names (the four consumer goods and the four service industries) on a 7-point scale with respect to their experience of the brand and their knowledge of the brand. Afterwards, the respondents were invited to evaluate two different websites of 
two different brands (one consumer good and one service industry website) on a 7-point Likert scale for different aspects of interactivity. The selection of the web site was at random. Questions were asked about four issues to measure perceived communication aspects of the website: (1) This website gives the opportunity to chat, mail, communicate; (2) the information of this website is up-to-date; (3) the website encourages visitors to communicate; and (4) the website is effective in gathering visitor's feedback. In addition, Song and Zinkhan's perceived control items of interactivity perceptions 2008 were measured. For perceived control, the navigation ('the amount of clicks necessary to go to the information') and the transmission of messages the website associated with the previous exchanges were measured. Furthermore, the perceived reaction to the perceived 'information' from the website was measured by asking about four items, for example, the effort/way/challenge/time to find the information on the website (Song and Zinkhan 2008). Afterwards, a more general attitude towards the website was measured using four items, whereby also the visual aspects of the website were taken into account (Song and Zinkhan 2008). Finally, we asked the respondents if they would recommend the website to their friends, and to give an overall rating of their 'global feelings' towards the website; both concepts were measured on a 7-point rating scale.

In Table 1 the research results of the second study of the preliminary research are given. The concepts measuring the different aspects of the website (interactivity, feelings towards the website and recommendation of the website) were each filled out by five subjects separately.

Table 1 shows that the consumer products 'Campina' and 'Jules Destrooper' have the highest scores for recommendation to friends, and that for these brands the general feeling towards the website and the different aspects of interactivity are relatively high compared to the other fast-moving consumer products - Evian and Brantano. However, knowledge and experience of Campina are significantly lower than for the other products, and for Jules Destrooper significantly higher than for the remaining products. Campina and Jules Destrooper also have significantly different scores with respect to knowledge ( $t$-value 3.348, d.f. 19; $p$-value 0.003) and experience ( $t$-value 4.046; d.f. 19; $p$-value 0.001). For the service industries, we have selected, according to the same method, Thomas Cook and De Lijn. Both brand names have high scores on interactivity, general feelings and recommendation.

Based on the results, we selected the fast-moving consumer brands "Campina" and "Jules Destrooper" and the service industry brands "Thomas Cook" and "De Lijn”. 


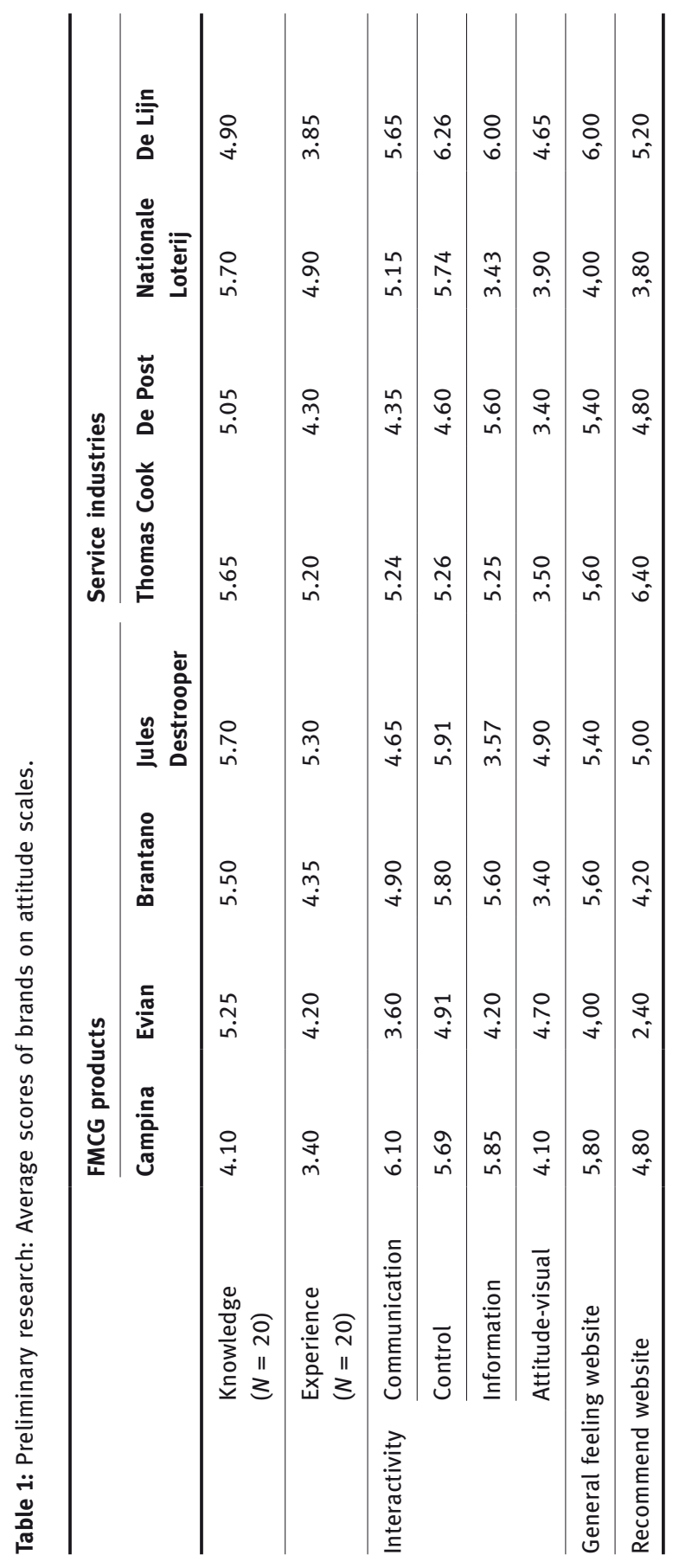




\subsection{Empirical research}

In the empirical study, the respondents were randomly assigned to one of the four different experimental groups. In each group, for the four different brand names four different advertisements involving four different touch points were given. To increase the external validity of our research, we have used existing advertisements involving the touch points, which had not been transmitted during the year before the empirical study was conducted. The research design could take place without interruption of the researcher or 'extra' effort by the respondents, thanks to the online software possibilities of Qualtrics. As discussed in the preliminary research, the advertising of the selected brand names was also spread across multiple media during a concentrated period in Belgium.

After viewing every advertisement (from the different touch point) the respondents were asked to fill out the questionnaire to evaluate the advertisement and the media-instrument. For example, the respondents in group I were first confronted with a TV commercial of Jules Destrooper, afterwards a radio spot of De Lijn, the website of Thomas Cook, and finally a print advertisement of Campina. For group II, the respondents were first confronted with a print advertisement of Thomas Cook, afterwards the website of Campina, a radio spot of Jules Destrooper, and finally a TV commercial of De Lijn. The aim of using the same brands in the different conditions was to compare directly both marketing communication techniques, on the one hand, and to control for brand preferences, knowledge of brand/product and other possible distortions caused by subjects' experience with the exhibited brands, on the other hand. We also carried out the measurements directly after the exposure of the advertisement to limit advertising carryover effects. In Figure 1, the research design of the

Figure 1: Research design of the empirical research.

\begin{tabular}{llll}
\hline Questionnaire1 & Questionnaire2 & Questionnaire3 & Questionnaire4 \\
\hline TV commercial & Print & Website & Radio \\
Jules Destrooper & Thomas Cook & De Lijn & Campina \\
\hline Radio & Website & Print & TV commercial \\
De Lijn & Campina & Jules Destrooper & Thomas Cook \\
\hline Website & Radio & TV commercial & Print \\
Thomas Cook & Jules Destrooper & Campina & De Lijn \\
\hline Print & TV commercial & Radio & Website \\
Campina & De Lijn & Thomas Cook & Jules Destrooper \\
\hline
\end{tabular}


four experimental groups with the four different touch points and brand names is shown.

\subsection{Sample}

Respondents were recruited from a consumer database available to the department of the Belgian university. Respondents were at least 18 years of age and were familiar with the touch points and had daily access to the internet. The respondents received an online invitation to join the survey. The invitation contained the URL link of the questionnaire and a note that if they were not interested in participating in this research, they were welcome to forward the link to someone else. The convenience sample in combination with a snow-ball method was necessary to collect sufficient respondents for the experimental research. The respondents were randomly exposed to one of the four experimental conditions. In total, 461 respondents started the questionnaire. Incomplete responses, questionnaires from the same IP-address and participants with the same responses for all questions were deleted (63.1\% of the responses). In total, 170 (completed) responses were used for our data analysis. Questionnaires 1 and 2 had 51 respondents each and questionnaires 3 and 4 had 34 respondents each. The average age of the respondents was 43.79 years (s.d. 15.34 years). No significant differences were found for age between the different experimental groups (F-value 0.096; d.f. 167; $p$-value 0.962). 57.6\% of the respondents were male; here, too, no significant differences between the experimental groups were found (chi-square 3.00; d.f. 1 ; $p$-value 0.392 ). $31.8 \%$ of the respondents had finished secondary school, $2.90 \%$ only primary school, $34.1 \%$ had gained a Bachelor degree and 32.2\% a Master's degree; here, too, no significant differences between the four different experimental groups were found (chi-square 9.058; d.f. 4; $p$-value 0.698). On average, the group of respondents consisted of $41.8 \%$ employees or workmen, $15.3 \%$ retired persons, $12.4 \%$ students, $4.1 \%$ housewives, $4.7 \%$ managers, $1.2 \%$ unemployed persons, $7.1 \%$ civil servants and $13.5 \%$ self-employed people. Here, too, no significant difference between the different experimental groups was found (chi-square 14.865; d.f. 21; $p$-value 0.830).

\subsection{Measurement and scaling}

Directly after viewing, or listening to, the advertisement, the respondents were asked to fill out the questionnaire. First, the different cognitive, emotional and 
conative concepts of the four different touch points (television, radio, print and website) were measured. The cognitive aspect 'the attitude towards the informative part of the advertisement' was measured using a 3-item 7-point Likert scale ("this advertisement is a good source for product information", "this advertisement provides relevant product information" and "this advertisement is a source of up-to-date product information") (Nagar, 2009). The results of the Cronbach's alpha analyses and the factor analyses indicated that we were allowed to investigate the items of the different aspects of attitude towards the advertisement 'informative part' as one concept (Cronbach's alpha score was 0.88 ; total variance explained $81.31 \%$; the concept is called 'information' in the Tables). The attention towards the advertisements was measured using a 3-item 7-point Likert scale based on Nagar 2009, that is, "the advertisement is attractive"; "I pay attention to the advertisement"; "this advertisement was eye-catching” (Cronbach's alpha 0.92; total variance explained 86.29\%, the concept is called 'attention' in the Tables). The persuasiveness of the advertisement was measured on a 3-item 7-point Likert scale based on Nagar 2009, for example, "this advertisement will help people change their attitude towards the product/brand"; "this advertisement will help people change the image of the advertising company"; "this advertisement will make the product/brand more appealing”. The Cronbach's alpha is 0.89, total variance explained $82.16 \%$; the concept is called 'persuasiveness'. The perceived entertainment value of the advertisements (an affective measurement of the touch point) was measured on a 3-item 7-point Likert scale, for example, "this advertisement is entertaining", "this advertisement is enjoyable" and "this advertisement is exciting” (Nagar 2009). The Cronbach's alpha is 0.89 , the total variance explained $82.07 \%$, and the concept is called 'entertainment'. For the concept 'credibility of the advertisements' we asked "Are important facts about the brand/product left out in this advertisement?", and for "clearness of the advertisements' we asked “Has this advertisement a clear message?” (Nagar 2009).

To measure attitude to the brand $(\mathrm{AB})$, we asked on a semantic differential scale of six items based on Spears and Singh 2004: "good/bad quality", "positive/negative”, “(not) attractive”, “(no) favor” (Cronbach’s alpha 0.942; variance explained $77.55 \%$ ). Afterwards, respondents were asked to give an indication of the possibility between 0 and $100 \%$ that they would purchase the brand/product after being exposed to the advertisement (Dahlén and Edenius 2007). Finally, the respondents gave a rank to the different touch points from most preferred to least preferred touch point based on Goldsmith and Lafferty 2002, and indicated gender, age category, education level and profession. At the end, we asked the respondents if they remembered the four different brand names in the advertisements (recall), and on the next page we asked to indicate 
the four different brand names (recognition), whereby, in total, 16 different brand names were shown (Hallward 2008; Goldsmith and Lafferty 2002). The average time to fill out the questionnaire was around 25 minutes.

\subsection{Research results}

Mixed models were used to estimate the different cognitive, affective and conative aspects of the different touch points of the different brands. Mixed models account for the repeated measures of the same participant by modelling the structure of association among the repeated measures. In a first stage we investigated whether the different touch points have to be interpreted for the different brands separately or if we were allowed to investigate on a touch point level. For every cognitive, affective and conative aspect, we estimated four different models. In the first two columns of Table 2, we analyzed the model for the different touch points (model (1)) and the model for the different brands (model (2)) separately. In the third model we estimated the model for the touch points and the brands, and in the fourth model the different interaction effects between the touch points and the brands were taken into account. In Table 2 the different log-likelihood fit values for the different models are given.

The research results of Table 2 show that the log-likelihood value of model (4) for the different concepts - whereby the main effects of the touch points and the brands and interaction effects of the touch points and the brands are taken into account - has the lowest scores. This means that the fit of model (4) should be better than the fit of the other models (see Table 2, the lowest fit scores of the different models for the different variables 'Fit (4-3)'). For almost all variables (except for entertainment and purchase intention) no significant differences were found between model (4) and model (3); this suggests that the interactions between the touch points and the brands are not significant.

By comparing model (3) with the model without the touch points (model (2) ('Fit (3-1)') or the model without the brands (model (2), 'Fit(3-2)'), we can conclude that the fit of the model will significantly decrease (reliability of < 0.001) when the touch points are not taken into account. However, the fit of the model does not significantly decrease (with the exception for the variable $\mathrm{AB}$ and $\mathrm{PI}$ ) if the brands are not taken into account. On the basis of this result we conclude that brands do not play a significant role when evaluating the different advertisements in our experiment. 


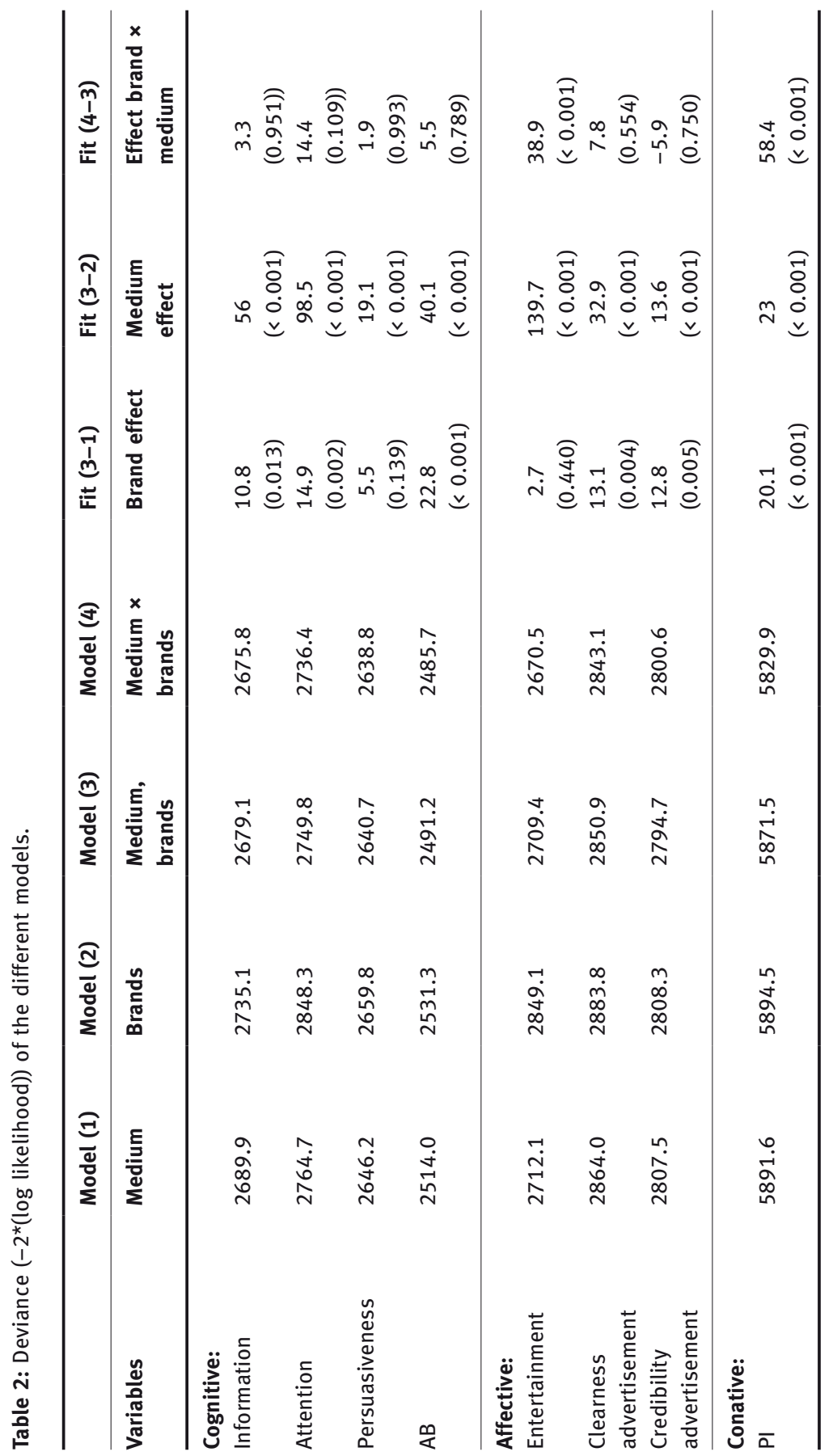




\subsection{Research question 1}

The research results found in Table 3 of the different cognitive, emotional and conative aspects of the different touch points give an answer to the first research question in which we investigate whether there are significant differences between the effectiveness of the different touch points. As we can see, the touch point 'television' scores significantly higher for the cognitive aspect 'attention' and the affective aspect 'entertainment', and attitude towards the brand. The results show that TV-commercials significantly influence the attitude of the customers towards the brand compared to other touch points. Furthermore, the results of Table 3 show that if the marketer of a brand wants to inform its customers about the product without entertainment, she or he must choose print advertisements. The 'information' and 'persuasiveness' of print advertising are significantly higher than for other touch points. Respondents have also indicated that the message of the print advertisements is significantly clearer compared to the website or the radio.

The conative aspect - purchasing intention - does not significantly differ between the different touch points. Also, the average percentages given by the respondents for purchase intention indicate that the probability is $29.46 \%$ that the respondent is willing to buy the product after seeing the print advertisement, for television the probability is $26.58 \%$, for websites $23.50 \%$, and for radio $22.98 \%$.

Subsequently, the respondents ranked the different touch points from most preferred to least preferred touch point. TV-commercials were by far the most preferred. $47.3 \%$ of the sample indicated TV commercials as the most preferred touch point, whereas for print advertisements $26.5 \%$ of the sample indicated them as most preferred. Radio had a score of $15.4 \%$ and websites of $11.2 \%$. The lowest scores were given to the media websites. $47.3 \%$ of the sample gave this media the lowest scores, followed by radio with $24.3 \%$, TV commercials with $15.4 \%$ and print advertisements with $12.9 \%$. The average ranking scores were: TV 2.03 (whereby 1 means highest rank and 4 lowest), print 2.29, radio 2.63 and internet 3.05. The Friedman test result has indicated significant differences (chi-square 59.069; d.f. 3, $N=167 ; p<0.001$ ). This suggests that TV commercials are the preferred touch point, and the non-traditional media channel 'the website' has, independently of the attitude scores, still a long way to go to be seen as an effective touch point. According to the general theory of effective communication (McLuhan 1964), the sender, i.e. the touch point, should match the preference of the receiver (TV commercials and not 'the website'). This result implies that for the second research question - the relative scores of the touch point - TV commercials as transmitter of the message are perhaps even more effective than those of the 'website'. 
Table 3: Estimates of the media model.

\begin{tabular}{lcccc}
\hline Variables & TV & Radio & Print & Website \\
\hline Cognitive: & & & & \\
Information & -0.4583 & -0.2978 & 0.4238 & 0.3324 \\
& $(<.0001)$ & $(<.0001)$ & $(<.0001)$ & $(<.0001)$ \\
Attention & 0.6448 & -0.2399 & 0.2660 & -0.6708 \\
& $(<.0001)$ & $(0.0030)$ & $(0.0010)$ & $(<.0001)$ \\
Persuasiveness & 0.1585 & -0.1440 & 0.2863 & -0.3008 \\
& $(0.0399)$ & $(0.0571)$ & $(0.0002)$ & $(<.0001)$ \\
AB & 0.3438 & -0.1861 & 0.2006 & -0.3583 \\
& $(<.0001)$ & $(0.0070)$ & $(0.0032)$ & $(<.0001)$ \\
\hline Affective: & & & & \\
Entertainment & 0.9306 & 0.08851 & -0.1848 & -0.8343 \\
& $(<0.001)$ & $(0.2608)$ & $(0.0200)$ & $(<0.001)$ \\
Clearness advertisement & 0.09576 & -0.1685 & 0.4611 & -0.3883 \\
& $(0.2697)$ & $(0.0512)$ & $(<0.001)$ & $(<0.001)$ \\
Credibility advertisement & 0.2575 & 0.07961 & -0.3341 & -0.00308 \\
& $(0.0031)$ & $(0.3515)$ & $(<0.001)$ & $(0.9707)$ \\
\hline Conative: & & & & \\
PI & 19.036 & -34.647 & 41.289 & -25.677 \\
& $(<0.001)$ & $(0.0070)$ & $(0.0032)$ & $(<0.001)$ \\
\hline
\end{tabular}

\subsection{Research question 2}

For the second research question, we have compared the awareness (recall and recognition) scores for the different touch points to analyze their effectiveness. In Table 4 an overview of the recall and recognition scores are given.

The estimates give an indication of the deviation from the 'global' average score.

The research results show that for the well-known brands (brands I and II, based on the preliminary research) the awareness scores are not significantly different between the different touch points. Only for the less known brands can significant differences be observed. The awareness scores for the less known service brand (brand III) is significantly higher for the advertisements on the radio, and the less known fast-moving consumer good (FMCG) (brand IV) has a significantly higher recall score with the TV commercial. This suggests that especially for unknown FMCG brands, television can stimulate remembrance of the brand name compared to the website. For brands of the service industry, the radio spot is significantly more effective to increase remembrance. 
Table 4: Recall and recognition scores for the different touch points and brands.

\begin{tabular}{|c|c|c|c|c|c|c|c|}
\hline & & \multirow{2}{*}{$\begin{array}{l}\text { TV } \\
N=51\end{array}$} & \multirow{2}{*}{$\begin{array}{l}\text { Print } \\
N=51\end{array}$} & \multirow{2}{*}{$\begin{array}{l}\text { Website } \\
N=34\end{array}$} & \multirow{2}{*}{$\begin{array}{l}\text { Radio } \\
N=34\end{array}$} & \multicolumn{2}{|c|}{ Test-value } \\
\hline & & & & & & $X^{2}$-value & $p$-value \\
\hline \multirow[t]{2}{*}{ Total } & Recall & $74.7 \%$ & $76.5 \%$ & $67.6 \%$ & $71.8 \%$ & 0.819 & 0.282 \\
\hline & Recognition & $83.5 \%$ & $90.6 \%$ & $85.3 \%$ & $81.8 \%$ & 5.909 & 0.116 \\
\hline \multirow[t]{2}{*}{ Brand I } & Recall & $80.4 \%$ & $64.7 \%$ & $79.4 \%$ & $82.4 \%{ }^{\star}$ & 5.06 & 0.168 \\
\hline & Recognition & $92.2 \% *$ & $76.5 \%$ & $91.2 \%$ & $88.2 \%$ & 6.50 & 0.090 \\
\hline \multirow[t]{2}{*}{ Brand II } & Recall & $72.5 \%$ & $80.4 \%$ * & $79.4 \%$ & $79.4 \%$ & 1.11 & 0.775 \\
\hline & Recognition & $86.3 \%$ & $80.4 \%$ & $88.2 \%{ }^{\star}$ & $88.2 \%{ }^{\star}$ & 1.49 & 0.686 \\
\hline \multirow[t]{2}{*}{ Brand III } & Recall & $51.0 \%$ & $76.5 \%$ & $85.3 \%$ & $94.1 \%$ & 2.37 & $<0.001$ \\
\hline & Recognition & $74.5 \%$ & $96.1 \%$ & $88.2 \%$ & $97.1 \%$ & 1.48 & 0.002 \\
\hline \multirow[t]{2}{*}{ Brand IV } & Recall & $72.5 \%$ & $66.7 \%$ & $38.2 \%$ & $67.6 \%$ & 1.15 & 0.009 \\
\hline & Recognition & $86.3 \%$ & $92.2 \%$ & $61.8 \%$ & $76.5 \%$ & 1.37 & 0.003 \\
\hline
\end{tabular}

Brand I: Jules Destrooper (FMCG, well known, lot of experience); Brand II: De Lijn (service, well known, lot of experience); Brand III: Thomas Cook (service, less known, less experience); Brand IV: Campina (FMCG, less known, less experience).

By calculating the average attitude scores for 'yes' or 'no' awareness, the results of Table 5 show that respondents remembering or recognizing the brand name have a significantly higher score for the different attitude concepts. This suggests that different attitude concepts towards the touch points can significantly influence the awareness scores. The average scores on the cognitive aspects 'informativeness', 'clear message', 'persuasiveness' and 'attention' are significantly higher for the respondents who remember or recognize the brand than for the respondents without that awareness. For the 'affective' aspects we have only found a significant difference for the 'entertainment' attitude; the respondents who were aware of the different brand names had significantly higher scores on the perceived entertainment value of the advertisement (the perceived credibility scores were only significantly different between respondents who recognized the brand and respondents who did not recognize the brand name). The intention to buy or use the brand was also significantly higher for those respondents who after the whole experiment were still aware of the different brand names which they had seen as compared to those who were not. This result suggests that the different aspects of attitude towards the advertisements can significantly influence the awareness score of the brand names. 


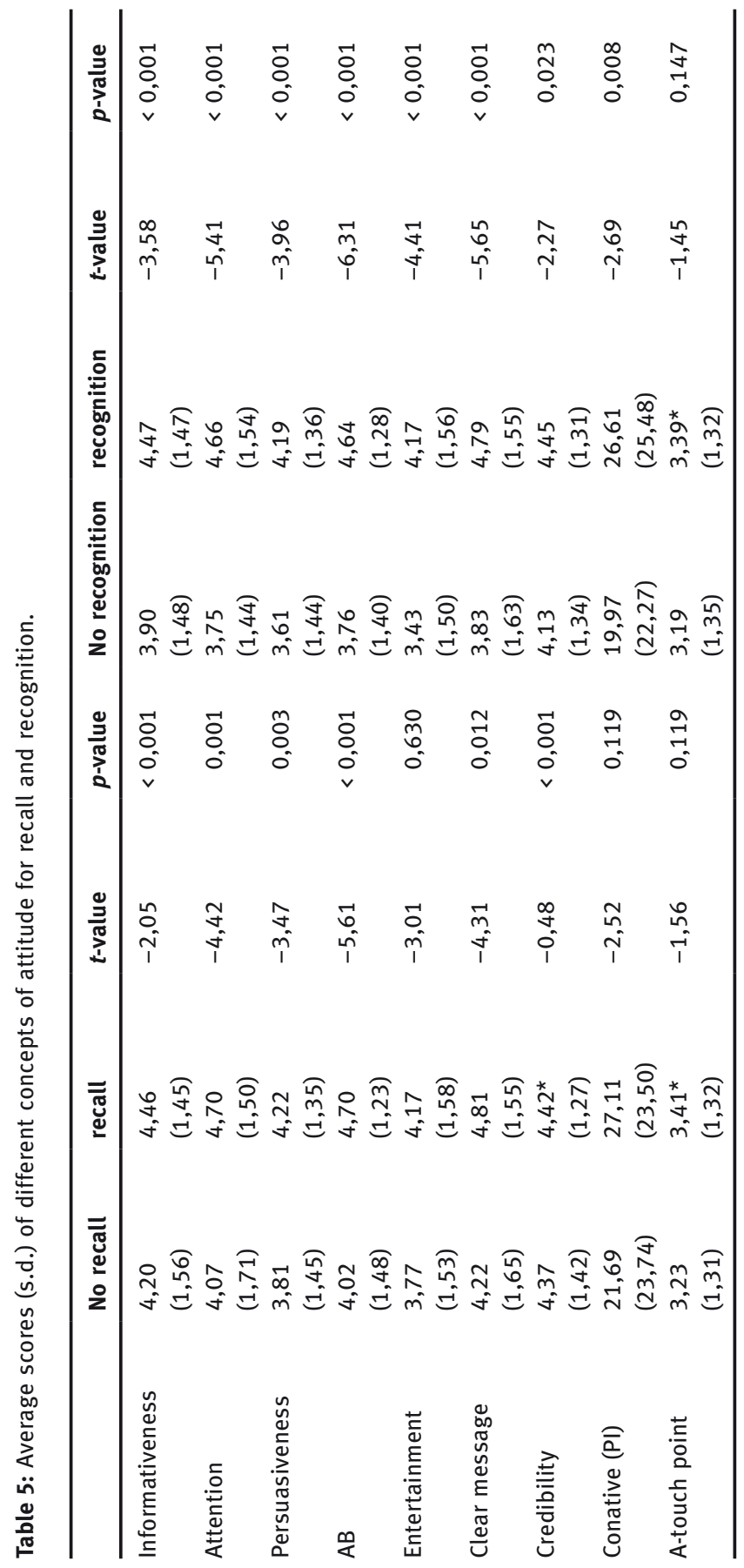




\section{Conclusions and discussion}

By comparing our findings with the communication objectives for the touch point planning (Dahlén and Edenius 2007; Danaher and Rossiter 2011; Naik and Raman 2003), we suggest the following conclusions. For creating specific brand experiences, our research results show that the traditional media channels, for example, television and print advertisements, have a bigger impact on the cognitive attitude toward the touch points, for instance, attention and persuasiveness. In addition, the entertainment content is significantly higher for the touch point television than for the other touch points. The touch point 'television' also creates a significantly higher score on the attitude towards the brand compared to the others. The significantly higher score on entertainment content also suggests that television as a touch point scores significantly higher on the communication objective 're-awaken or stimulate positive emotions to stimulate a relationship between the customer and the brand'. These results are in accordance with the "uses and gratifications" theory of Katz, Haas, and Gurevitch 1973, indicating that communication receivers have needs (e.g., information, knowledge, understanding) that can be met by different communication channels. In addition, our results also corroborate the findings of the survey by Goldsmith and Lafferty 2002, which indicates that, in general, consumers liked TV and print advertisements more than the advertisements on the radio and the internet.

According to our results, print advertisements generate the highest score for the general attitude, 'information' of the message and the clearness of the message of the advertisement. This result suggests that print advertisements are best used to influence customer thinking about the core value proposition of the brand. The results also indicate that TV-commercials and secondly print advertisements are the 'preferred' touch points to radio or internet. The fact that increasingly TV viewers engage in many activities, including glancing at a magazine, while watching TV ("multifunctional") suggests that both touch points can be 'effective' at the same time. Earlier research also shows the synergy effect of both media (Lin et al. 2013; Naik and Raman 2003). However, the literature provides limited empirical and theoretical knowledge of cross-media synergy.

In our research the role of the different touch points in creating value in the consumer brand relation could not be measured as such. However, a first external valid indication of the degree to which a touch point has the ability to influence the different attitudes of consumers for the same brands was investigated. In doing so a distinction was made for well-known/unknown and FMCG and services. Our findings show that the overall results for the website (the 
internet) as touch points compared to the others are relatively negative. The preliminary research results show that the interactivity level of the websites is relatively high but the low 'entertainment' and the 'attention' scores suggest that as a touch point they cannot compete with TV commercials. The awareness scores also underline these findings; especially the recall scores for the FMCG are relatively low compared to the other touch points. These findings suggest that marketing managers should invest in creating internet sites with the qualities that make the site more entertaining and likeable.

In conclusion, the high scores in our research for the TV commercial compared to the other touch points is notable, which indicates that respondents preferred a TV commercial as a contact between the brand or organization and the respondent. Websites have, on the other hand, a very low score. This is consistent with the limited literature which has analyzed these issues: Goldsmith and Lafferty 2002 and Danaher and Rossiter 2011 found that consumers appear to like print advertisements and TV significantly more than the advertisements they recalled seeing on the internet. The more robust methodological setup of our research, therefore, corroborates earlier findings based on more ad hoc methodologies.

Further research could usefully include other touch points which we did not study such as internet banner advertisements, word-of mouth, displays in supermarkets, billboards and cinema commercials.

A general limitation of the cross-sectional approach of our study based on data of a (representative) convenience sample of four brands is that it does not allow cause and effect interference. However, the important advantage of this experimental setting is the high degree of internal control it generates, which makes it possible to compare the different touch points at a given point in time. Previous literature relying on protocol data or self-rate surveys (Goldsmith and Lafferty 2002; Smith, Gopalakrishna, and Chaterjee 2006) does not ensure that respondents have actually viewed all the different touch points, and the robustness of the conclusions about the relative effectiveness of these touch points is, therefore, limited. Our research design does, however, require forced exposure to the advertisements to control for brand interferences, for example, experience, knowledge and/or brand preference. This, obviously, limits the extent to which our results can be generalized. Further research that explicitly takes account of the fact that most of the media channels can only be effective if the consumer is watching a screen (TV commercial, mobile/smart phone or computer), listening (radio spot) or reading (print advertisement) is, therefore, needed. 


\section{References}

Bruner II, G. C., \& Kumar, A. 2000. Web commercials and advertising hierarchy-of-effects. Journal of Advertising Research, 40(1/2), 35-42.

Dahlén, M., \& Edenius, M. 2007. When is advertising advertising? Comparing responses to non-traditional and traditional advertising media. Journal of Current Issues and Research in Advertising, 29(1), 33-42.

Danaher, P. J., \& Rossiter, J. R. 2011. Comparing perceptions of marketing communication channels. European Journal of Marketing, 45(1/2), 6-42.

Dubow, J. S. 1994. Point of view: Recall revisited: Recall redux. Journal of Advertising Research, 34(3), 92-108.

Goldsmith, R. E., \& Lafferty, B. A. 2002. Consumer response to web sites and their influence on advertising effectiveness. Internet Research: Electronic Networking Applications and Policy, 12(4), 318-328.

Green, H., \& Elgin, B. 2001. Do e-ads have a future. The race is on to find ways to increase internet advertising's effectiveness. Business Week, 22 January, 46-50.

Hallward, J. 2008. "Make measurable what is not so": Consumer mix modelling for the evolving media world. Journal of Advertising Research, 48(3), 339-351.

Homer, P. M. 1990. The mediating role of attitude toward the ad: Some additional evidence. Journal of Marketing Research, 17(1), 78-86.

Jenkinson, A. 2007. Evolutionary implications for touchpoint planning as a result of neuroscience: A practical fusion of database marketing and advertising. Database Marketing \& Customer Strategy Management, 14, 164-185.

Karson, E. J., McCloy, S. D., \& Bonner, P. G. 2006. An examination of consumers' attitude and beliefs towards web site advertising. Journal of Current Issues and Research in Advertising, 28(2), 77-91.

Katz, E., Haas, H., \& Gurevitch, M. 1973. On the use of the mass media for important things. American Sociological Review, 38(4), 164-181.

Lin, C., Venkataraman, S., \& Jap, S. 2013. Media multiplexing behavior: Implications for targeting and media planning. Marketing Science, 32(2), 310-324.

MacKenzie, S. B., Lutz, R. J., \& Belch, G. E. 1986. The role of attitude toward the advertisements as a mediator of advertising effectiveness: A test of competing explanations. Journal of Marketing Research, 23, 130-143.

McKenzie, K. E., \& Royne, M. B. 2009. Defining and understanding communications planning: A current assessment and an exploratory study. Journal of Promotion Management, 15(3), 341-356.

McLuhan, M. 1964. Understanding Media: The Extensions of Man; $1^{\text {st }}$ Ed. McGraw Hill, NY; reissued by MIT Press, 1994.

Moorman, C. 2013. The CMO survey: Social media spending by marketers to skyrocket. Retrieved May 8, 2014 from http://www.fuqua.duke.edu/news_events/news-releases/ cmo-survey-results-feb-2013/\#.U2jjWIGSzTp.

Nagar, K. 2009. Advertising effectiveness in different media: A comparison of web and television advertising. IIMB Management Review, 21(3), 245-260.

Naik, P. A., \& Raman, K. 2003. Understanding the impact of synergy in multimedia communications. Journal of Marketing Research, XL, 375-388. 
Newhagen, J. E., Corders, J. W., \& Levy, M. R. 1995. Nightly@nbc.com: Audience scope and the perception of interactivity in viewer mail on the internet. Journal of Communications, 45(3), 164-175.

Schultz, D. E., Cole, B., \& Bailey, S. 2004. Implementing the 'connect the dots' approach to marketing communication. International Journal of Advertising, 23, 455-477.

Smith, T. M., Gopalakrishna S., \& Chaterjee, R. 2006. A three-stage model of integrated marketing communications at the marketing-sales interface. Journal of Marketing Research, 43(4), 564-579.

Song, J. H., \& Zinkhan, G. M. 2008. Determinants of perceived web site interactivity. Journal of Marketing, 72(2), 99-113.

Spears, N., \& Singh, S. 2004. Measuring attitude toward the brand and purchase intentions. Journal of Current Issues \& Research in Advertising, 26(2), 53-66.

Yoon, S. J., \& Kim, J. H. 2001. 'Is the internet more effective than traditional media? Factors affecting the choice of media'. Journal of Advertising Research, 41(6), 53-60. 
Copyright of Communications: The European Journal of Communication Research is the property of De Gruyter and its content may not be copied or emailed to multiple sites or posted to a listserv without the copyright holder's express written permission. However, users may print, download, or email articles for individual use. 